\section{Will we need proton therapy in the future?}

\section{E.Pedroni, Paul Scherrer Institute, Division of Radiation Medicine, CH-5232 Villigen PSI, Switzerland}

C ancer is the second major cause of death (after cardiovascular diseases) in the developed countries. Cure from cancer can be achieved nowadays for about $45 \%$ of all cancer patients using currently available therapeutic strategies: surgery, radiation therapy and chemotherapy. For about $2 / 3$ of the patients the disease is still well localized within a specific region of the body at the time when the patient is confronted with the diagnosis of cancer. For these patients the chances of cure using a local therapy, surgery or radiation therapy, are reasonably good.

Whenever possible, a radical surgical excision of the disease is the preferred therapeutic choice. The earlier the diagnosis and the smaller the tumor, the better the chances for a good therapeutic outcome. In this context screening plays an important role in the early detection of the disease. Surgery is the most successful therapy since it contributes $22 \%$ to the overall cure rate. Radiation therapy (RT) is the second most effective modality. RT is used with curative intent when the tumor is inoperable but is still well localized in a specific region of the body. RT contributes $12 \%$ to the cure rate alone and $6 \%$ in combination with surgery. When the disease has already spread in the whole body (with distant metastases) the chances of cure are correspondingly lower. Chemotherapy is then used with the intent to eliminate the diffused cancer cells. Chemotherapy and the other remaining modalities account for the last $5 \%$ of the total cure rate.

The largest effort in cancer research is undertaken today by big pharmaceutical companies on new developments based on modern biological sciences. The most promising ones are expected today from genetic technologies. Like other systemic therapies, these methods will face the problem of unwanted side effects of the drugs, which are inevitably spread through the whole body. The huge number of cancer cells involved in a solid tumor and the difficulty of transporting the drug into the center of the tumor are the major problems of the utilization of drugs for the elimination of advanced solid tu- mors. For these reasons the new biological methods still aim (like chemotherapy) in the first place at the inactivation of isolated cancer cells or small tumor volumes (metastasis) with the intent to control the microscopic spread of the disease.

It is the experts' opinion that for the foreseeable future, surgery and radiation therapy will continue to play a major role in the control of primary solid tumors. While waiting for an eventual breakthrough from genetic technologies, it is necessary to continue to improve the established local methods, surgery and radiotherapy. It is also important to note that the different types of therapy are not necessarily exclusive and are often used in a complementary way. Many recent successes in cancer management are in fact based on the combined use of different modalities. About $2 / 3$ of all cancer patients receive radiation therapy alone or in combination with other modalities. A reduction of the toxicity of a therapy modality automatically improves the tolerance of the others in a combined treatment.

Improvements in RT were achieved in the past by using advanced treatment techniques (for example conformal radiation therapy) and/or by using unusual types of radiation (like external beam therapy with protons or light ions). In this report we will discuss primarily proton therapy. Many of the presented arguments apply as well to therapy with heavier ions.

\section{The rationale for the use of} protons for therapy

Protons have a well-defined range of penetration in materials (the range depends on the selected initial energy of the beam), and they show a pronounced dose maximum in the region where the beam stops. This region is called the Bragg peak. Fig. 1 shows the dose deposition of a mono-energetic proton beam as a function of the depth in water. The dose profile of a proton beam de- posited with energy modulation, the socalled spread out Bragg peak (SOBP), is compared in the same picture with the corresponding dose profile of clinically used photons, which show a characteristic exponential fall-off of the dose with increasing depth.

The possibility to use the Bragg peak as a practical tool for the localization of the dose in depth has been known for more than 50 years (first proposed in 1946 by Robert R.Wilson). At that time modern computer technology for treatment planning and the precise physical description of the patient's anatomy provided by computer tomography (CT) were not available. Technological advances in both these areas allow us today to take full advantage of the inherent precision of this method.

The main advantage of proton therapy is expected from the superior capability to confine the dose on the target volume and so to reduce the dose burden to the surrounding healthy tissues. The major object of comparison for proton (and ion) therapy is clearly conventional therapy with photons. Protons are expected to produce superior results for the treatment of large tumors of complex shape, where a significant reduction of the dose outside of the target volume is clinically desirable. Compared to photons, one can expect to achieve with protons a general reduction of the integral dose deposited outside of

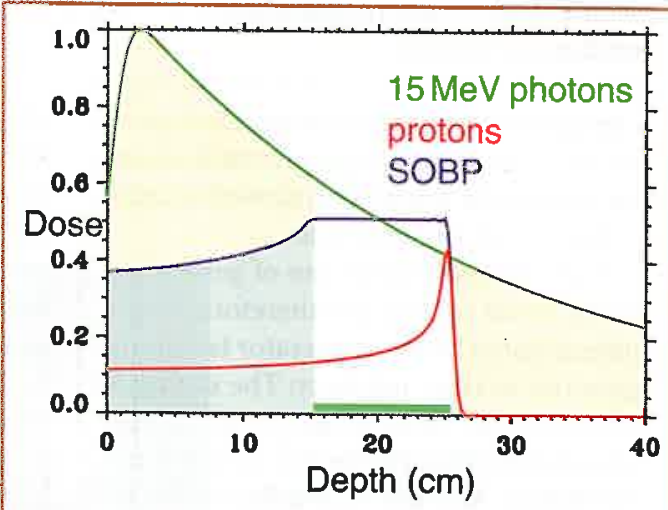

Fig. 1: Comparison of the depth dose profiles of proton and photon beams. The photon dose falls off exponentially with depth (green curve). A mono-energetic proton beam is characterized by the presence of the Bragg peak in the region where the protons stop (red profile). Through the superposition (blue curve) of many proton beams of different residual range it is possible to deposit a homogenous dose (SOBP) in the region of the tumor (in this case from 15 to $25 \mathrm{~cm}$ depth). One recognizes from the picture the potential of dose sparing of the protons in the entrance and exit region of the beam (the unnecessary dose is painted in pale blue and light yellow). 
the target volume by a factor of 2 or more. This dose sparing could be relevant in many situations, for example for radiation therapy of pediatric tumors. Children surviving cancer often present with severe consequences of the treatment in adulthoad, like reduced intelligence after brain irradiation or an abnormal growth after treatments covering part of the skeleton. By using protons one can expect to reduce these deleterious effects. It is very important to note that the clinical goal of proton therapy is not only to increase patient survival but also to achieve a better quality of live after the treatment. From the radiobiological point of view protons behave like photons in conventional therapy. The experience acquired in the hospitals on the tolerances of the different organs and the response to radiation of the various types of cancers can be directly applied to protons.

The major disadvantage of proton therapy is the large size of the accelerator and of the beam lines needed for the transport of the beam. The maximum proton energy needed for applying proton therapy on deep-seated tumors is of the order of 230 $250 \mathrm{MeV}$. Because of the resultant magnetic rigidity, the beam lines are heavy and the accelerators are rather large $(4$ to $7 \mathrm{~m}$ diameter) compared to electron linacs (a $1 \mathrm{~m}$ long accelerator rotating on a gantry on a diameter of about $3 \mathrm{~m}$ ). It is therefore simply the question of size and costs, which hinders proton therapy to be more widely spread in the hospitals.

We should mention here briefly also ion therapy. Concerning the improved localization of the dose heavier ions behave similarly (to some extent better) to protons, but the magnetic rigidity needed for the transport of the beam is by a factor of 3 higher than with protons. The accelerator and beam lines are correspondingly larger and therefore even more expensive than for proton therapy. The most important issue is however the difference in the radiobiological behavior of these highLET beams. We mention here the inhibition of spontaneous repair of radiation damages affecting both the cancer and healthy tissue cells. The high LET could bring advantages for the treatment of certain types of radio-resistant tumors but could also be a disadvantage for many other treatments with respect to a possibly higher rate of late complications of normal tissues. Ion therapy represents a very interesting addition to photon and proton therapy. However more scientific evidence of the merits, based on clinical results for selected indications is required, before thinking of an eventual diffusion of this method on a commercial basis. Ion therapy is still a matter of research as opposed to proton therapy, which could become soon a business issue.

\section{The recent developments in} conventional therapy: Photon IMRT The success of RT in general is based on the ability to confine the dose delivery within a small region of the body (the target volume, which must contain the whole extent of the visible and invisible tumor). Ideally one would like to deposit only the necessary dose inside the target volume and zero dose outside. In practice we are faced with strong physical and technical limitations.

The state of the art in conventional radiation therapy is based on the use of very compact electron linacs mounted in the head of a rotating gantry. The photon beam is produced through the Bremstrahlung of the electrons impinging on a metallic target (the photon source). Through the use of a rotating gantry the beam can be directed onto the supine patient from several directions. The localization of the dose in depth is then achieved through the superposition of several converging beams.

The use of sophisticated beam delivery techniques, the implementation of computer technology and the information gained with modern diagnostic techniques (CT, MRI and PET) have been at the origin of the progress achieved in RT in the last two decades. These modern methods aim to shape the dose in all 3 dimensions to conform precisely to the individual shape of the target volume (conformal radiation therapy).

The use of dynamic computer-controlled multi-leaf collimators offers here new additional possibilities. The most interesting is to apply the dose with a non-uniform distribution of photon fluence for each of the constituent dose fields. The superposition of intentionally non-homogeneously shaped dose distributions can produce a resultant dose distribution of superior quality (with a higher degree of conformity, especially in the case of target volumes with concavities). This new approach is called intensity-modulated radiotherapy (IMRT). The optimization of the delivery of radiation using multiple beam ports is a typical "inverse problem" with a strong analogy to computer tomography (CT). With CT one uses multiple projections

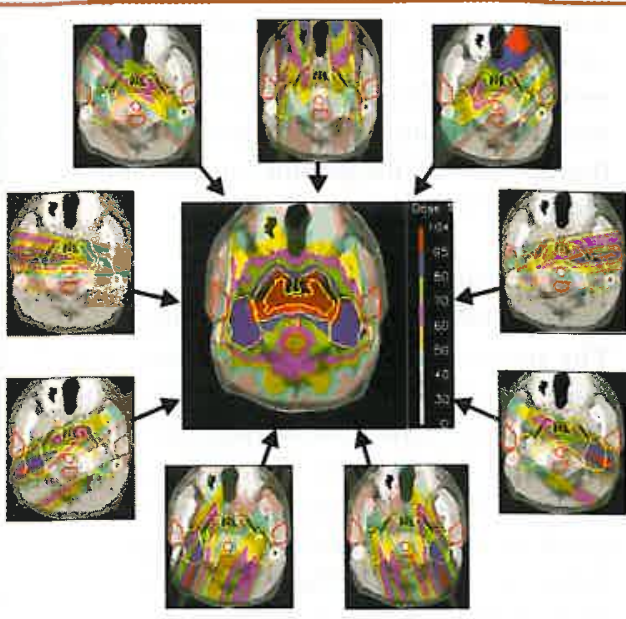

Fig 2: An example of intensity modulated treatment planning with photons. Through the addition of 9 fields it is possible to construct a highly conformal dose distribution with good dose sparing in the region of the brain stem (courtesy of T. Lomax, PSI).

(from many angles) to reconstruct complex density images. With IMRT one uses complex dose projections to produce more idealized dose distributions. Tomotherapy is a similar approach to IMRT, where the analogy with CT is at the closest also from the point of the beam delivery, since the beam is applied here slice by slice with gantry rotation in-between.

Fig. 2 shows an IMRT example with the dose distribution for a nasopharyngeal tumor. The calculation has been done with the treatment-planning package of PSI. The dose is delivered using 9 photon fields. Each field is applied with modulation of the photon flux. One recognizes immediately that the optimization algorithm avoids in this example to send photons towards the brain stem in each of the fields. The dose delivered to this sensitive organ gets reduced considerably in this way. The availability of a large amount of degrees of freedom in the beam delivery and the strength of the mathematical methods make it possible to produce very satisfying dose distributions, shaped in all 3 dimensions to conform precisely to the target volume.

Many of the professionals working in the hospitals are now convinced that photon IMRT will become soon established enough to make proton therapy unnecessary. IMRT is certainly an improvement for radiation therapy and a big challenge for all centers investigating the potential of proton therapy in the world. The fact that the majority of the medical physicists working in the hospitals are very excited about IMRT and not so much about pro- 
ton therapy, is due to the fact that they consider proton therapy out of their personal reach. The question for the people working in proton therapy is: can proton therapy take up the gauntlet and do better than IMRT?

\section{The equivalent developments for} protons: RIMPT and beam scanning The most elegant, flexible and efficient method for providing inverse planning with protons (IMPT) is by magnetic beam scanning. Protons are charged particle beams. The beam can be steered very quickly in the patient's body by magnetic deflection. This could be a more practical alternative to multileaf collimators. Fig.3 shows the basic principles of the beam scanning technology. The dose distribution of a proton pencil beam is characterized by a shallow entrance dose (the plateau region) followed by a well-localized peak at the end of the range (a dose hot spot of typically $1 \mathrm{~cm}$ size). The pencil beam is scanned in the lateral direction by magnetic deflection in the beam line ahead of the patient. The modulation in depth is achieved by changing the range of the protons dynamically. A high conformity of the dose can be achieved by changing the exposure time and the position of each pencil beam individually under computer control. This method provides individually shaped dose distributions, which are conformed in all 3 dimensions, even when using a single beam direction (conformal therapy with variable modulation of the range). The required dose homogeneity inside the target volume is thus achieved separately for each constituent field.

Beam scanning is also well suited for the delivery of the dose, when the optimization is performed simultaneously on many fields, in order to take full advantage of all possible degrees of freedom. The constituent fields are then not necessarily homogenous but the result of the superposition of the dose is. This can improve dose sparing on some critical organs. In our terminology this technique was originally called simultaneous dose optimization. In order to make the analogy with conventional photon IMRT more obvious, we often call-improperly- this technique IMPT (intensity modulated proton therapy). The difference to IMRT is that with proton beam scanning, we can adjust also the proton range. Each parameter, proton flux (dosage), proton range and beam direction (gantry angle) can be independently varied in relation to the point of the Bragg peak inside the target volume. We

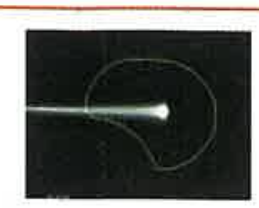

Single beam...

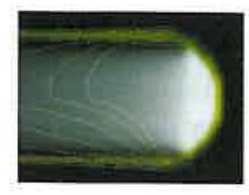

+ scanning in depth

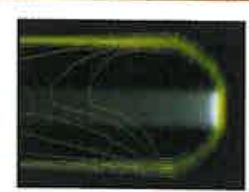

(lateral scanning

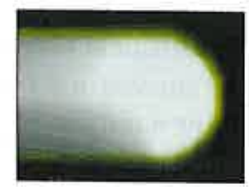

$=3 d$ conformed dose)
Fig 3: Basic principle used for beam scanning with protons. Through the delivery of individual proton pencil beams one can shape the distribution of the dose in three-dimensions at wish directly under computer control.

should therefore call the method more properly Range-Intensity Modulated Therapy (RIMPT) to underline the added freedom to control directly the dose localization in depth.

Fig. 4 shows an example of RIMPT. With only 4 modulated fields one can deliver a highly conformal dose to the primary target and a reduced dose to the affected lymph nodes (the secondary target) with a maximal sparing of the organs at risk (brain stem and parotid glands). With beam scanning, RIMPT can be calculated and delivered just under computer control without the need for any patient specific hardware. The main difference compared to photon IMRT is obviously the absence of the "dose bath" to the whole brain as depicted in figure 2.

\section{The status of proton therapy in the world}

The realization at the beginning of the 90 's of the first hospital-based proton therapy facility of the world at the Loma Linda University Medical Center (LLUMC) in the Los Angeles area is a major milestone in the history of proton therapy. The components of the facility - a dedicated synchrotron feeding three proton gantries and two horizontal beam lines - were designed, realized and tested first at Fermilab (as a spin-off product from basic physics research) before being transferred to the LLUMC. Patient treatments were started in 1990. Up to now more than 5000 have been treated in this hospital with protons. Loma Linda is working since several years showing a steady improvement of patient throughput, which has recently reached the capability to treat close to 1000 patients per year. The big merit of this University is that it has paved the way for the development of proton therapy not only on scientific goals, but also on the basis of commercial criteria and routine use in a hospital environment.

Fig.5 shows as an example the layout of the North East Proton Therapy Center (NPTC) in Boston, which is the next hospital-based proton facility of the USA. This facility has been delivered by a European company and is expected to go into operation at the beginning of the next year at the Massachusetts General Hospital in Boston. The accelerator is in this case a cyclotron.

In Japan, there are several facilities already installed or under construction. The centers are in Chiba (for carbon ions), Kashiwa, Tsukuba, Shizuoka Prefecture, Wakasa Bay (for protons) and Hyogo (for protons and ions). These facilities are designed, delivered and operated by major Japanese industrial companies.

Concerning possible hospital-based solutions, in Europe we are still in the phase of discussions.

The time needed for the preparation of the patient before the treatment is usually a significant fraction of the total treatment time. This is why all new dedicated proton facilities are designed with one accelerator for the delivery of the beam sequentially into several treatment rooms. All new proton facilities use nowadays a rotating beam line for the beam delivery, the socalled isocentric gantry. All commercial facilities presently available are using a beam delivery method based on passive scattering. The idea is to produce with distant

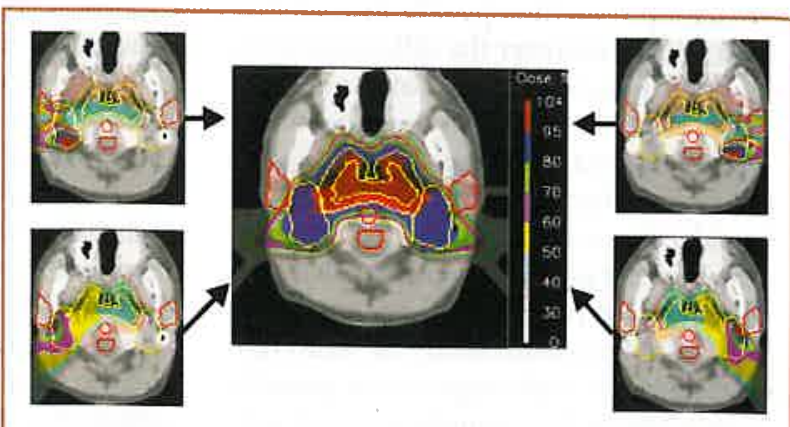

Fig 4: Example of intensity modulated therapy with protons. A high degree of conformity is achieved using a low number of dose fields. The advantage compared with photons is the general reduction of dose burden outside of the target volume (courtesy of T.Lomax, PSI) 
scatterers a homogeneous flux of protons in the solid angle covering the target volume. The lateral conformation of the dose is then achieved by using individually shaped collimators. The conformation of the dose in depth is controlled by the use of range-shifter wheels and individually shaped compensators. The method has been developed in close analogy to conventional therapy with photons. The passive scattering technique requires a long throw of the beam on the gantry head (the distance between the last bending magnet and the patient). The proton gantries dedicated to scattering are for this reason very large, with a diameter of the rotating structure of the order of 10-12 meters. The passive scattering technique relies almost completely on the use of several beam modifiers machined individually for each patient and each dose field, and consequently the manipulation of the equipment is cumbersome and time consuming. The passive method is also difficult to upgrade to RIMPT.

The new aproach chosen at PSI (A GSI for ion therapy): Active beam scanning

\section{The delivery of proton therapy by beam scanning}

At present the proton facility of PSI in Switzerland is the only one capable of delivering proton therapy on a very compact gantry using a dynamic beam scanning technique (spot scanning). GSI (Darmstadt Germany) has developed a similar beam delivery system (raster scanning) in a horizontal beam line for the delivery of scanned ion beam therapy (with carbon

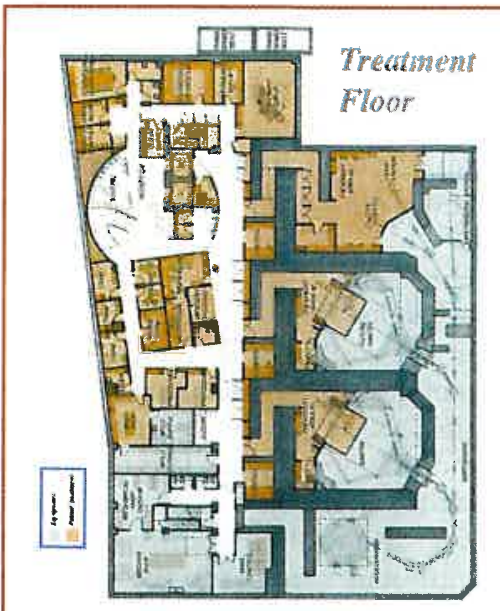

Fig 5: Layout of the NPTC proton therapy facility of the

Massachusetts General Hospital in Boston U.S.A. The facility has been delivered by a Belgian company (courtesy of J. Flanz, MGH).

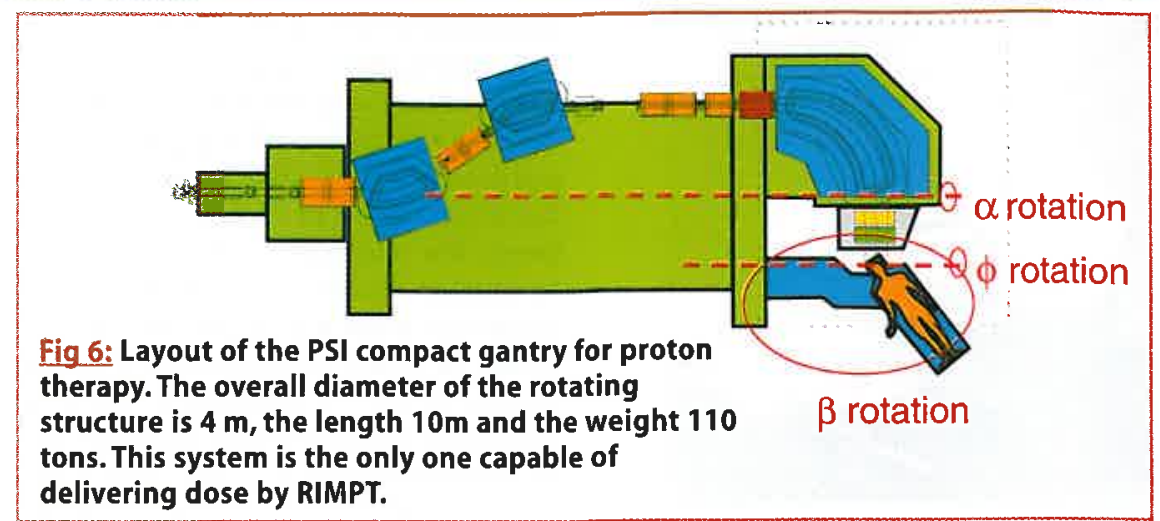

ions). If Europe is last in providing dedicated facilities to the hospitals, it is first in the related technological developments.

Figure 6 shows the layout of the compact eccentric gantry of PSI. The gantry is dedicated to beam scanning and is the only system currently capable of delivering proton RIMPT. The lateral scan of the beam is performed in one direction magnetically before bending the beam towards the patient. The space between the bending magnet and patient can then be reduced essentially to zero. The other lateral scan motion is obtained by moving the patient table. An eccentric mounting of the patient table on the gantry reduces further the gantry diameter, which at only $4 \mathrm{~m}$ is the most compact of all present designs. The goal was here to achieve a radius of the rotating structure similar to the conventional photon gantries in the hospitals. Fig.7 shows a picture of the PSI treatment room. The system at PSI has been operational since 1996 for patient treatments. The typical throughput of the facility is about 2 patients per hour. This comprises the administration of several fields ( 3 on the average) in the same session. The change of gantry angle is performed without the need of intervention of the personnel inside the treatment room.

The positive experience gained with this system has now convinced the PSI directorate of the necessity to provide a dedicated medical accelerator for the project, in order to provide all year round beam for medical treatments, and in order to commercialize the developed technology. For the last goal PSI plans the development of a second gantry, an improved version of the present design, with the patient table mounted at the isocenter. The second gantry is foreseen as a more general and more user friendly instrument for use in the hospitals.

The interest in the spot scanning technology is steadily increasing in the world, mainly due to the challenge posed by photon IMRT. All dedicated proton facilities are now planning to develop beam scanning in addition to scattering on the long throw gantries.

The debate within proton therapy: beam delivery by scattering or scanning? or both?

Beam delivery methods for IMRT with protons will be needed soon for the competition with photon IMRT. One could think eventually to use dynamic multileaf collimators on top of the passive scattering technique (with compensators and range shifter wheels). In this case the modulation of the range would remain conhowever, with active control of the local modulation of the range, beam scanning is probably the only reasonable solution. The next question is then, why not choose for dedicated proton facilities industrial solutions based only on scanning and consequently take advantage of small compact gantries?

The major point advanced in favor of the scattering method, is the higher sensitivity of the dynamic beam scanning technique with respect to dose errors due to organ motion compared to passive scattering. This is the Achilles' heel of all dynamic beam delivery methods, including IMRT with photons. The best solution would be to try to improve the speed of scanning to allow for multiple repaintings of the target volume. This is the subject of studies for the next gantry of PSI (gantry 2). Another possible solution is to trigger the beam within a phase interval of the breathing cycle of the patient (this technique has been developed by our colleagues in the Japanese hadron therapy centers). PSI is also investigating the use of small magnetic sensors for monitoring the position of moving tumors during treatment (project TULOC). All these solutions are under consideration for the next development phase of the PSI project.

The medical doctors seem at the moment to be very reluctant to abandon the stant. For the delivery of true RIMPT, 


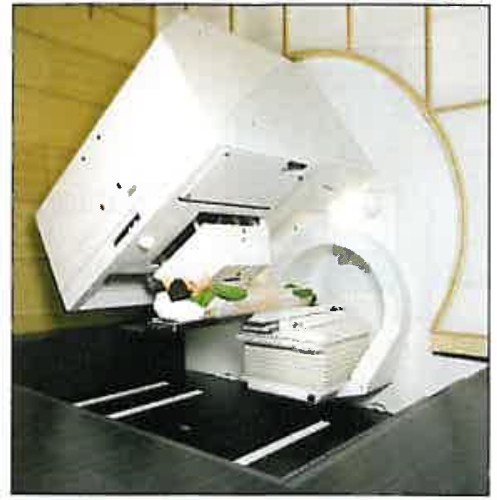

Fig 7: Photograph of the PSI proton treatment room with the head of the compact gantry dedicated to proton beam scannning.

traditional passive scattering method. In principle nothing forbids to perform the scanning with a broad beam on a very coarse grid to produce a flat field of homogeneous dose and to carve out the edges of the field with collimators and compensators, as this is done with the passive scattering technique. By using a broad beam we could increase the grid spacing of the scan and so afford to repaint the target several times. This approach could simulate scattering well enough to convince people to replace scattering by scanning without the fear of loosing something. To this purpose we are improving the design of our beam delivery system to allow for much larger variations of the beam size.

Gantry systems providing both beam delivery techniques, scanning and scattering, are expected to be complex, large and expensive. In the long range the development of more advanced beam scanning techniques capable of replacing passive scattering is probably a necessary condition if we want proton therapy to be economically more competitive with photons.

\section{Discussion}

Radiotherapy represents an important instrument in the fight against cancer in a field of continuous evolution. In conventional therapy we expect to be able to observe in the near future a significant progress using very advanced beam delivery techniques with photons (IMRT). Similar more advanced developments are in principle possible also with charged particle beams (RIMPT). The physical advantages of protons applied with new beam delivery techniques are expected to produce superior results. This is why we believe that beam scanning will be soon a necessary option for any new proton and ion beam therapy center.

Just from physical arguments we should always obtain with protons superior dose distributions compared to photons (with marginal exceptions like the skin-dose or the lateral fall-off in very deep-seated tumors). The selection of the patients could be quickly decided just on the basis of treatment planning. If proton therapy would be widely available with a similar routine as for the photons, it would be technically possible to offer an (objectively) better treatment to a very large number of patients.

Let us consider now the question of the costs.

The equipment of a proton therapy center is more expensive, of the order of $25-40 \mathrm{M} \$$ (depending on the number of treatment rooms used in the facility), than the 3-5 millions needed for the corresponding units in a modern radiotherapy department. The amount of trained personnel needed for the delivery of a sophisticated radiation treatment is however expected to be very similar with protons or photons. The personnel costs for running the facility are expected at the end to be the major expenditure in the budget for the total lifetime of a dedicated proton facility.

Very rough estimates indicate that the costs for a high-tech proton treatment could be about the double of the costs for an average conventional treatment. The costs of general radiotherapy are similar to surgery, but much cheaper then chemotherapy and genetic technologies (chemotherapy is generally used with less chances of success than radiotherapy even if it is more expensive). The difference in costs between proton and photon therapy is very modest in relation to the general medical costs of handling cancer as such, independently of the results. If the treatment with protons avoids some treatment complication (with all the related expenses over years to cope with them), protons can be justified purely on the basis of economical arguments. Our conviction is that the additional costs for proton therapy are worthy of consideration.

The attempt to quantify scientifically the benefits is one of the main goals of the clinical trials being performed in the research centers offering proton therapy for research purposes, like ours. The process of assessing results in the treatment of cancer is a difficult one and requires many years. The group at the Harvard cyclotron and MGH/Boston has delivered most of the existing scientific evidence in favor of the protons. We mention here the very good results for tumors close to the base of the scull and treatments of eye melanoma (at PSI alone more than 3000 eye patients have been treated for this indication). World wide the experience of using charged particle beams has reached already a total of about 30000 patients. However, with the new beam scanning methods the experience is still very limited.

If proton therapy centers would be widely available offering proton therapy on the basis of a well-established knowhow, the majority of cancer patients would probably prefer to be treated with this method without waiting for further scientific results of clinical studies. Everybody would choose for himself a treatment with a lower burden to the healthy tissues compared to photons, even if the photon dose is declared to be below the tolerance level for complications. In view of the growing wealth and requirements on quality of life in western society, it is not an unrealistic scenario to assume that many people would probably be ready to pay for the difference or to put pressure on their health insurance companies.

Thanks to the pioneering work done at scientific institutions, a lot is known on the use of charged particle beams. The feasibility of further improvements has been also demonstrated at research centers.

Proton therapy and ion therapy is now in a transition characterized by the imminent availability of many new dedicated hospital facilities in Japan and U.S.A.

It is reasonable to assume that something similar will happen also in Europe. This could be even through private clinics financed by profit oriented organizations with the sole purpose of earning money. Even this would be good news for cancer patients. To say the least, we would not have missed another important opportunity for spin-offs from basic research in physics.

\section{Acknowledgments}

The author would like to thank the colleagues of the team for radiation medicine of PSI for their invaluable contribution to the proton therapy project. This report has been written on behalf of this group. Many thanks go also to the PSI directorate for the continuous support to the development of radiation medicine. We acknowledge also the whole proton therapy community, represented by the Proton Therapy Co-operative Group (PTCOG), for the very constructive and collaborative spirit, which has characterized this organization for many years. 